\title{
THE CONCENTRIC SHELL METHOD FOR RELATIVISTIC STAR CLUSTERS
}

\author{
E. D. FACKERELL \\ Dept. of Applied Mathematics, University of Sydney, Australia
}

\begin{abstract}
The analytic aspects of the Campbell-Hénon method of concentric spherical shells are generalized for application to relativistic spherically symmetric star clusters.
\end{abstract}

\section{Introduction}

Hoyle and Fowler (1967) gave considerable impetus to the study of the properties of relativistic star clusters when they introduced their relativistic star cluster model for quasistellar sources. In this model the redshift is gravitational in origin, since the emitting region is postulated to be at the centre of a highly relativistic star cluster. The main difficulty with this model is to find reasonable models of relativistic star clusters which are stable and yet which have large central redshifts $\left(z_{c}\right)$. Ipser $(1969 \mathrm{~b})$ found that wide classes of reasonable models of relativistic spherically symmetric collisionless star clusters become unstable against radial perturbations at values of the central redshift greater than about 0.5. Using a refinement of Ipser's method, Fackerell (1970) showed that a relativistic polytropic star cluster model with a core-halo structure was unstable for $z_{c} \gtrsim 0.73$. Because of these results interest in the Hoyle-Fowler model waned after 1970 .

However, in a recent publication Horwitz et al. (1974) have claimed, on the basis of a thermodynamic stability criterion, that there exist stable truncated isothermal relativistic star clusters with values of $z_{c}$ as high as 4 . These clusters have a pronounced core-halo structure, and so the analysis of the dynamical stability of these models requires the introduction of more powerful methods than those available at present, since Ipser's methods are usually inconclusive for models with pronounced core-halo structures (Ipser, 1969a, b). A further reason why it is desirable to develop new methods for the analysis of the dynamical stability of relativistic clusters is that all existing methods require that the equilibrium distribution function should be a monotonic decreasing function of energy (Ipser and Thorne, 1968). Another problem which is as yet intractable and which nevertheless is of considerable interest is the investigation of the effect on a cluster of a central black hole.

In Newtonian theory the most significant method developed to date for the analysis of the stability against radial disturbances of collisionless spherically symmetric stellar systems is the concentric shell method due to Campbell (1962) and developed for full numerical application by Hénon (1964. 1967). Hénon has demonstrated the power of this method in his investigation of the stability of a wide class of generalized polytropic models (Hénon, 1973). A compact summary of the method has been given by Janin (1972). 
The purpose of this paper is to begin the development of a general relativistic version of the Campbell-Hénon method. This requires the solution of three problems, namely, (1) the analysis of the motion of a spherical shell with a given 'equation of state' in a spherically symmetric gravitational field (possibly due to a black hole), (2) the determination of the 'equation of state' of a shell of collisionless particles, and (3) the determination of the configuration that arises when two collisionless shells pass through one another.

\section{Motion of a Spherical Shell in General Relativity}

The motion of a spherical surface layer in general relativity has been investigated by a number of authors. Israel $(1966,1967)$ investigated the motion of a pressure-free shell collapsing under its own self gravitation. Chase (1970) extended Israel's formalism to deal with a charged shell with internal pressure moving in the combined field due to itself and to an internal central charged mass. The equation of motion that we require may be deduced from Chase's results by setting all charges equal to zero.

The spherical shell, which we denote by $\Sigma$, has the parametric equations

$$
\begin{aligned}
& r=R(\tau), \\
& t_{+}=T_{+}(\tau),
\end{aligned}
$$

and

$$
t_{-}=T_{-}(\tau),
$$

where $\tau$ is the proper time on the shell, $t_{+}$and $t_{-}$are the Schwarzschild times on the exterior and the interior of $\Sigma$ and $r$ is the radius of the shell in Schwarzschild coordinates. Note that, while $r$ is continuous across the shell, the Schwarzschild time is discontinuous across the shell. If the mass interior or the shell is $m_{1}$ and the mass of the whole system is $m_{2}$ (both in geometrized units), the equation of motion of the shell is given by

$$
\left(\dot{R}^{2} / c^{2}+1-2 m_{1} / R\right)^{1 / 2}-\left(\dot{R}^{2} / c^{2}+1-2 m_{2} / R\right)^{1 / 2}=\frac{4 \pi G}{c^{2}} R \sigma,
$$

where

$$
\dot{R}=\mathrm{d} R / \mathrm{d} \tau
$$

and $\sigma$ is the surface energy density of the shell. If the surface pressure of the shell is denoted by $p$, the surface energy tensor $S_{a b}$ of the shell is given by

$$
S_{a b}=\left(\sigma+\frac{p}{c^{2}}\right) U_{a} U_{b}+\frac{p}{c^{2}} g_{a b} \quad(a, b=0,1,2),
$$

where $g_{a b}$ is the intrinsic metric tensor of the shell and $U_{a}$ is the intrinsic velocity of an observer moving with the shell. If we take our intrinsic coordinates $\xi^{a}$ on the shell as

$$
\left(\xi^{0}, \xi^{1}, \xi^{2}\right)=(\tau, \theta, \phi)
$$


we find that

and

$$
\left(U^{0}, U^{1}, U^{2}\right)=(c, 0,0)
$$

$$
\left(g_{a b}\right)=\operatorname{diag}\left(-c^{2}, R^{2}(\tau), R^{2}(\tau) \sin ^{2} \theta\right) .
$$

$S_{a b}$ satisfies the conservation law

$$
S_{; b}^{a b}=0
$$

which in our case becomes

$$
\mathrm{d}\left(R^{2} \sigma\right) / \mathrm{d} \tau+2 R \dot{R} \frac{p}{c^{2}}=0 .
$$

The Schwarzschild times on the two sides of $\Sigma$ may be determined from the differential equations

$$
\frac{\mathrm{d} T_{+}}{\mathrm{d} \tau}=\left(1-2 m_{2} / R\right)^{-1}\left(\dot{R}^{2} / c^{2}+1-2 m_{2} / R\right)^{1 / 2}
$$

and

$$
\frac{\mathrm{d} T_{-}}{\mathrm{d} \tau}=\left(1-2 m_{1} / R\right)^{-1}\left(\dot{R}^{2} / c^{2}+1-2 m_{1} / R\right)^{1 / 2}
$$

Equations (4), (5), (11) and (12) are sufficient to determine the motion of the shell provided $\sigma$ and hence $p$ are given as functions of $R$.

\section{Equation of State of a Shell of Collisionless Particles}

The kinetic theory of surface layers has been developed in general relativity by Voorhees (1972) whose results may be stated as follows. The surface energy tensor is given by

$$
S_{a b}=\int \frac{1}{\mu} f p_{a} p_{b} \mathrm{~d} v_{(p)},
$$

where $f$ is the distribution function in the surface layer, $\mu$ is the conserved rest mass of a particle with intrinsic momentum $p_{a}$ and $\mathrm{d} v_{(p)}$ is the natural element of volume in the momentum space associated with the surface layer. The distribution function $f$ is to be a suitable function of the constants of the motion of a particle in the surface layer. These constants can be obtained from the intrinsic Hamilton-Jacobi equation

$$
g^{a b} \frac{\partial S}{\partial \xi^{a}} \frac{\partial S}{\partial \xi^{b}}=-\mu^{2} c^{2}
$$

which in our case becomes

$$
\frac{1}{c^{2}}\left(\frac{\partial S}{\partial \tau}\right)^{2}-\frac{1}{R^{2}(\tau)}\left[\left(\frac{\partial S}{\partial \theta}\right)^{2}+\frac{1}{\sin ^{2} \theta}\left(\frac{\partial S}{\partial \phi}\right)^{2}\right]=\mu^{2} c^{2}
$$


so that

$$
\begin{aligned}
& p_{0}=-c\left[\mu^{2} c^{2}+\alpha^{2} / R^{2}(\tau)\right]^{1 / 2} \\
& p_{1}= \pm\left[\alpha^{2}-J^{2} / \sin ^{2} \theta\right]^{1 / 2} \\
& p_{2}=J
\end{aligned}
$$

where $J$ is the axial component of angular momentum and $\alpha^{2}$ is the squared angular momentum.

If all the particles have the same rest mass $\mu_{0}$ and the same squared angular momentum $\alpha_{0}^{2}$, a suitable distribution function is

$$
f=\frac{1}{2 \pi^{2}} A \delta\left(\mu-\mu_{0}\right) \delta\left(\alpha^{2}-\alpha_{0}^{2}\right)
$$

where the constant $A$ is in fact the total number of particles in the shell, since the intrinsic particle flux vector

$$
N_{a}=\int \frac{1}{\mu} f p_{a} \mathrm{~d} v_{(p)}
$$

has the sole surviving proper component

$$
N_{(0)}=A /\left(4 \pi R^{2}\right) \text {. }
$$

From Equations (13) and (19) we find that

$$
\sigma=\frac{A}{4 \pi R^{2} c}\left[\mu_{0}^{2} c^{2}+\alpha_{0}^{2} / R^{2}\right]^{1 / 2}
$$

and

$$
p=\frac{A \alpha_{0}^{2} c}{8 \pi R^{4}}\left[\mu_{0}^{2} c^{2}+\alpha_{0}^{2} / R^{2}\right]^{-1 / 2} .
$$

These expressions differ from those given by Papapetrou and Hamoui (1968). However, they are entirely compatible with Gerlach's results on the motion of a thin shell (Gerlach, 1970) so we are compelled to reject the results of Papapetrou and Hamoui together with those of Urbantke (1972) who followed their derivation.

If we substitute Equation (22) into Equation (4) and solve for $\dot{R}^{2}$ we obtain the equation of motion in the form

$$
\frac{1}{c^{2}}\left(\frac{\mathrm{d} R}{\mathrm{~d} \tau}\right)^{2}=-1+\frac{m_{1}+m_{2}}{R}+\frac{\left(m_{2}-m_{1}\right)^{2}}{K^{2}}+\frac{1}{4} \frac{K^{2}}{R^{2}}
$$

where the variable $K$ is defined by

$$
K=\frac{G A}{c^{3}}\left(\mu_{0}^{2} c^{2}+\alpha_{0}^{2} / R^{2}\right)^{1 / 2} .
$$




\section{The Crossing of Two Shells}

We now consider the problem of determining the configuration that results when two collisionless spherical shells pass through each other at radius $R$ say. Let the velocity of the inner shell just before crossing be specified by $\dot{R}_{1}^{-}=\mathrm{d} R_{1} / \mathrm{d} \tau_{1}$, where $\tau_{1}$ is the proper time on this shell and the derivative is evaluated just before crossing. Let the total number of particles in this shell be $A_{1}$, let their common rest mass be $\mu_{1}$, let their common angular momentum have magnitude $\alpha_{1}$ and let the variable $K_{1}$ be defined by

$$
K_{1}=\frac{G A_{1}}{c^{3}}\left(\mu_{1}^{2} c^{2}+\alpha_{1}^{2} / R^{2}\right)^{1 / 2} .
$$

Let $\dot{R}_{2}^{-}, \tau_{2}, A_{2}, \mu_{2}, \alpha_{2}$ and $K_{2}$ have similar meanings for the outer shell. Let the Schwarzschild mass of the region interior to the inner shell be $m_{1}$, let the Schwarzschild mass of the region between the two shells be $m_{2}$ before crossing takes place and let the Schwarzschild mass of the total system be $m_{3}$. We need to determine the Schwarzschild mass $m_{4}$ of the region between the shells after crossing occurs together with the new velocity $\dot{R}_{1}^{+}$of the new outer shell and the new velocity $\dot{R}_{2}^{+}$of the new inner shell.

Because $A_{1}, \mu_{1}, \alpha_{1}, A_{2}, \mu_{2}, \alpha_{2}$ and therefore $K_{1}$ and $K_{2}$ are all conserved we obtain the two relations

$$
\left(\dot{R}_{1}^{+} / c\right)^{2}=-1+\frac{m_{3}+m_{4}}{R}+\frac{\left(m_{3}-m_{4}\right)^{2}}{K_{1}^{2}}+\frac{1}{4} \frac{K_{1}^{2}}{R^{2}}
$$

and

$$
\left(\dot{R}_{2}^{+} / c\right)^{2}=-1+\frac{m_{4}+m_{1}}{R}+\frac{\left(m_{4}-m_{1}\right)^{2}}{K_{2}^{2}}+\frac{1}{4} \frac{K_{2}^{2}}{R^{2}}
$$

between the three unknowns $\dot{R}_{1}^{+}, \dot{R}_{2}^{+}$and $m_{4}$. A third relation comes from the fact that the relative velocity of the shells before crossing is equal to their relative velocity after crossing. We find that

$$
\begin{array}{r}
{\left[\left(\dot{R}_{1}^{+} / c\right)^{2}+1-2 m_{4} / R\right]^{1 / 2}\left[\left(\dot{R}_{2}^{+} / c\right)^{2}+1-2 m_{4} / R\right]^{1 / 2}-\dot{R}_{1}^{+} \dot{R}_{2}^{+} / c^{2}=} \\
=\left(1-2 m_{4} / R\right) Y,
\end{array}
$$

where $Y$ is defined by

$$
\begin{aligned}
Y=\left\{[ ( \dot { R } _ { 1 } ^ { - } / c ) ^ { 2 } + 1 - 2 m _ { 2 } / R ] ^ { 1 / 2 } \left[\left(\dot{R}_{2}^{-} / c\right)^{2}\right.\right. & \left.+1-2 m_{2} / R\right]^{1 / 2}- \\
& \left.-\dot{R}_{1}^{-} \dot{R}_{2}^{-} / c^{2}\right\} /\left(1-2 m_{2} / R\right) .
\end{aligned}
$$

A tedious algebraic manipulation yields the elegant result

$$
m_{4}=m_{1}+m_{3}-m_{2}+Y K_{1} K_{2} / R,
$$

which shows that the mass energy of a given shell is not conserved during the crossing of that shell with some other shell. The values of $\dot{R}_{1}^{+}$and $\dot{R}_{2}^{+}$may then be determined from Equations (27) and (28), the ambiguities of sign being resolved by the fact that we require $\dot{R}_{1}^{+}>\dot{R}_{2}^{+}$and we also require that (29) should be satisfied. 


\section{Problems in Integration of the Equations}

It is clear that the problem of numerical integration of the basic equations for relativistic shells will be considerably more difficult than the corresponding Newtonian problem. The difficulties arise principally because of the fact that each shell needs its own time coordinate and because the $\dot{R}_{i}$ are discontinuous at the crossing of shells, together with the fact that the mass-energy of a given shell is also discontinuous at shell crossings. The last two facts require that the precise spatial location of each shell crossing has to be determined, which will add considerably to computing time. The problem of different times for each shell may be overcome by the expedient of slicing space-time into sections determined by taking constant Schwarzschild time between shells, but it needs to be noted that the difference in Schwarzschild time between successive slices will not be constant in between different shells. Finally we note that the collapse of a shell inside its event horizon does not cause any serious computing problem since we have used proper time on the various shells as our basic time coordinates. Once the innermost shell has begun an irreversible journey towards its event horizon it may be ignored in further computation, apart from the fact that its mass is then to be added to the mass of the central black hole.

\section{Discussion}

Two main areas of research will be opened up by the development of a successful numerical integration procedure for relativistic shells. First, it will be possible to investigate the full non-linear stability of the truncated isothermal clusters introduced by Zel'dovich and Podurets (1965) and Fackerell (1966). This will enable a decisive answer to be given to the claim by Horwitz et al. (1974) that there exist stable clusters with high central redshifts. Second, the interesting problem of the effect of a central black hole on the evolution of a spherical cluster will be numerically analyzable. Both of these problems are being pursued by Mr. K. G. Suffern at the University of Sydney.

\section{Acknowledgement}

I wish to acknowledge the helpful and constructive criticism of K. G. Suffern during the course of this work.

\section{References}

Campbell, P. M.: 1962, Proc. Nat. Acad. Sci. 48, 1993.

Chase, J. E. : 1970, Nuovo Cimento 67B, 136.

Fackerell, E. D.: 1966, 'Relativistic Stellar Dynamics', University of Sydney (Ph.D. Thesis).

Fackerell, E. D.: 1970, Astrophys. J. 160, 859.

Gerlach, U. H.: 1970, Phys. Rev. Letters 25, 1771.

Hénon, M.: 1964, Ann. Astrophys. 27, 83.

Hénon, M.: 1967, 14e Colloque de Liège, p. 243.

Hénon, M.: 1973, Astron. Astrophys. 24, 229.

Horwitz, G., Katz, J., and Klapisch, M.: 1974. 'Relativistic Star Clusters with High Gravitational Redshifts?', Racah Institute of Physics. Hebrew University of Jerusalem (preprint). 
Hoyle, F. and Fowler, W. A.: 1967, Nature 213, 373.

Ipser, J. R.: 1969a, Astrophys. J. 156, 509.

Ipser, J. R.: 1969b, Astrophys. J. 158, 17.

Ipser, J. R. and Thorne, K. S. : 1968, Astrophys. J. 154, 251.

Israel, W.: 1966, Nuovo Cimento 44B, 1.

Israel, W.: 1967, Phys. Rev. 153, 1388.

Janin, G.: 1972, in M. Lecar (ed.), Gravitational N-Body Problem (IAU Colloq. 10), D. Reidel Publ. Co., Dordrecht-Holland, p. 311.

Papapetrou, A. and Hamoui, A.: 1968, Ann. Inst. H. Poincaré A9, 179.

Urbantke, H.: 1972, Acta Phys. Aust. 35, 1.

Voorhees, B. H.: 1972, Phys. Rev. D5, 2413.

Zel'dovich, Y. B. and Podurets, M. A.: 1965, Astron. Zh. 43, 963. 